\title{
Continuidad y cambio en la política exterior chilena en el gobierno de Sebastián Piñera (2010-2014)
}

\author{
Foreign policy: continuity and change during Sebas- \\ tián Piñera's Administration (2010-2014)
}

\author{
Sebastián Briones \\ Andrés Dockendorff **
}

\section{Resumen}

El presente trabajo examina la política exterior del gobierno de Sebastián Piñera. Para ello, se sopesa en tres partes la importancia de un cambio de coalición gobernante en Chile después de varias décadas. Primero, se examina someramente la bibliografía relevante en materia de diplomacia presidencial relacionada con el continente, América Latina y Chile. A continuación, se analiza la inserción internacional del país a la luz de las nociones de integración y regionalismo abierto. En tercer lugar, se describen los hitos de la política exterior del gobierno de Piñera en función de su programa, las relaciones comerciales, los ámbitos regional y vecinal y la seguridad internacional. Finalmente se pondera el peso de esa política exterior en la medida en que preserva y modifica la posición de Chile en el panorama global y regional.

Palabras Clave: política exterior- regionalismo abierto- Alianza del Pacífico- relaciones comerciales- seguridad internacional

\footnotetext{
Profesor de la Academia Nacional de Estudios Políticos y Estratégicos (ANEPE).

** Profesor Asociado, Facultad de Ciencias Políticas y Administración Pública, Universidad Central. adockendorff@gmail.com Recibido el 10 de agosto de 2014; aceptado el 3 de marzo de 2015.
} 


\section{Abstract}

This paper examines foreign policy during Sebastián Piñera's administration. The significance of a change in the ruling coalition after several decades is analyzed in three different parts. First, the paper surveys the relevant literature on presidential diplomacy in the continent, Latin America, and Chile. Then, it analyses the country's international insertion according to the concepts of integration and open regionalism. Thirdly, it describes foreign policy milestones of the Piñera administration examining his program, as well as commercial relations, regional and neighborhood policy and international security. Finally, it ponders the significance of that foreign policy in terms of the extent in which the country's position in the global arena was modified or preserved.

KeYwords: foreign policy- open regionalism- Pacific Alliance- commercial relations-international security 


\section{INTRODUCCIÓN}

La alternancia dejaba planteada la interrogante de si traería aparejada un cambio en la agenda internacional de Chile. Por una parte, resultaba algo esperable que un gobierno de derecha tuviese visiones o alianzas diferentes de aquellas de sus antecesores. Pero por otra, la política exterior aplicada durante los gobiernos de la coalición de centro izquierda, a través de la firma de acuerdos de libre comercio con los principales países y bloques comerciales a que pertenecía el país, resultaba congruente con los postulados de la derecha. Los bloques comerciales menos flexibles, como el Mercado Común del Sur (MERCOSUR), de los que Chile no es parte, no parecían generar mayor interés en el nuevo gobierno. La participación de Chile en otras instancias regionales o globales tampoco experimentó grandes cambios.

En el presente artículo se sostiene que en la política exterior del gobierno de Sebastián Piñera se observan dos fenómenos fundamentales. Uno predominante es la preservación de la política de acuerdos comerciales suscritos con el mundo, que marca una línea de continuidad. Esto también se observa en la participación de Chile en tareas de seguridad internacional. El otro fenómeno, que algunos pueden calificar de ruptura y otros de profundización del estilo: la creación y participación activa de Chile en la Alianza del Pacífico. No obstante, en la medida en que lo anterior pueda calificarse de cambio dependerá de dónde se sitúe la línea de base del análisis, toda vez que los países aplican criterios diferentes respecto de la orientación de las alternativas de integración actualmente existentes.

Para abordar la dinámica de continuidad y cambio de la política internacional chilena en el primer gobierno de derecha en 50 años, se pasará revista a alguna bibliografía sobre la diplomacia presidencial. En una segunda sección se revisará la situación actual en materia de integración y cómo ella puede dar luces sobre el tipo de fenómeno que implica, por ejemplo, la Alianza del Pacífico. En una tercera sección se revisarán la agenda de política exterior de Piñera contenida en su programa de gobierno, las relaciones comerciales, regionales, vecinales y su participación en instancias de cooperación en seguridad internacional. Si bien se trata de un simple ejercicio de racconto, que corre el riesgo de incurrir en omisiones diacrónicas importantes, se esbozan algunas conclusiones sobre el periodo analizado y las principales líneas de continuidad y de ruptura parcial en la agenda de política exterior.

\section{LA POLÍTICA EXTERIOR DE LOS PRESIDENTES}

La bibliografía relativa a la política exterior de los presidentes es de larga data, pero buena parte de ella está centrada en el caso de Estados Unidos y las posibilidades de extrapolar esa realidad son limitadas. Teniendo presente la 
importancia que tendría para el público masivo, se atribuyó un 20 por ciento de la aprobación presidencial a temas de política exterior en el caso de Estados Unidos (MacKuen, 1983). Otros autores acotan el impacto de la política exterior señalando que es un factor de corto plazo. Aunque la política exterior podría transitoriamente impulsar la popularidad presidencial, suelen predominar los temas internos (Marra, Ostrom y Simon, 1990). McAvoy incorpora esta bibliografía, así como mucha anterior sobre estabilidad y cambio en la aprobación presidencial. Por un lado, confirma la visión de Marra y otros (1990), pero contiene reservas sobre la influencia de los factores internacionales. Así, se darían diferencias apreciables en sus efectos en la opinión pública, según la región del mundo en que están envueltos los intereses nacionales (McAvoy, 2006). En otra línea de análisis, se ha comprobado también un creciente involucramiento de la presidencia en la promoción y firma de acuerdos internacionales (Caruson y Farrar-Myers, 2007)

Dentro de América Latina, Raúl Bernal-Meza constata el incremento de la diplomacia presidencial durante la presidencia de Cardoso. En palabras del autor: «(...) o estilo da política exterior seja pragmático, com um marcado 'presidencialismo' (ou diplomacia presidencial), durante o período de Cardoso.» (Bernal Meza, 2002: 59). Con todo, se advierte que ya era una práctica habitual: «Historicamente o Brasil usou a 'diplomacia presidêncial' somente nas suas relações bilaterais com seus principais sócios (Argentina, Paraguai, Uruguai e Estados Unidos)» (Bernal Meza, 2002: 59). Otros, como Alcides Costa consideran que esta forma de política exterior predomina sobre otros: «(...) e apesar do decisivo exercício de uma intensa diplomacia presidencial e do ainda escasso envolvimento de outras instâncias sócio-políticas no debate» (Costa, 1999: 63).

En casos como el de Venezuela, las características personales de algunos presidentes, como Hugo Chávez, permitirían explicar el papel de la presidencia en lo relacionado con la política exterior (Fermín, 2012). Según Anabella Busso, la experiencia argentina ha permitido demostrar el impacto de las condiciones políticas internas en el cambio o statu quo de la política exterior de dicho país. De ese modo, situaciones como las crisis económicas y políticas experimentadas por el país, o la polarización en torno al modelo de desarrollo a seguir, han provocado la discontinuidad de la política exterior de Argentina desde el retorno a la democracia en 1983 (Busso, 2014).

En lo que respecta al caso de Chile, la bibliografía existente sugiere que dados ciertos acuerdos a nivel de élite que permiten perfilar la política exterior, la diplomacia presidencial cumpliría un papel de articuladora de intereses más ampliamente compartidos, aunque no necesariamente por toda la sociedad: «Em que pese clara inclinação da elite chilena pela integração do país a economias desenvolvidas, assistiu-se também 
no Chile a um proceso de tensionamento político-partidário de acordos comerciais» (Onuki y Oliveira, 2006: 148). Morandé ya había advertido ese rasgo en el pasado: «In general, the individual/idiosyncratic level of analysis plays a subordinate role to state- and systemic-level factors in the orientation and formulation of Chilean foreign policy» (Morandé, 2003: 262).

\section{EL CONTEXTO INMEDIATO: INTEGRACIÓN Y REGIONALISMO}

No será sino hasta el empalme de los procesos de democratización en América Latina y la aplicación de políticas de desregulación y apertura económica en los años ochenta y comienzos de los noventa, que adquiere prominencia un esquema particular de integración que reconocía la diversidad de la región: el «regionalismo abierto».

¿En qué situación se encuentra el esquema del «regionalismo abierto" más de dos décadas después de que comenzara a aplicarse? Para algunos autores, ya a fines de la primera década del siglo XXI, el regionalismo abierto como expresión del nuevo regionalismo latinoamericano parece hacer cerrado un ciclo (Sanahuja, 2007). Ello, dado un contexto en que los procesos de integración chocan con la existencia de diferentes estrategias de desarrollo entre los países de la región, así como de políticas internas divergentes (Phillips, 2003; Gardini, 2010; Grugel y
Riggirozzi, 2012; Malamud y Gardini, 2012; Nolte y Wehner, 2013).

En ese contexto, en la actualidad surgiría lo que se ha denominado «regionalismo post-liberal», que constituye una agenda menos próxima del aperturismo y la libertad comercial características del «regionalismo abierto» (Riggirozzi, 2012; Sanahuja, 2012; Riggirozzi y Tussie, 2012; Chodor y McCarthy-Jones, 2013). Lo anterior sería reflejo de la búsqueda de un nuevo modo de relacionamiento entre países de la región y el mercado global post Consenso de Washington (Grugel, Riggirozzi y Thirkell-White, 2008).

El Gráfico 1 representa las áreas temáticas en que se han desarrollado los diversos acuerdos internacionales. La cobertura o profundidad exacta de cada acuerdo pueden ser debatibles. (Según Oelsner, 2013: 15, el MERCOSUR, por ejemplo, dista mucho de siquiera hacerle honor a su nombre como «mercado único»). Pero es importante diferenciar entre la dimensión de las áreas temáticas consideradas por cada acuerdo y la intensidad o estado de la relación. Los esquemas regionales de integración no han logrado cubrir ambos aspectos, además del alcance regional (número de países que adhieren a cada esquema). 
Gráfico 1: Diversos acuerdos internacionales según el estado de la relación y el ámbito de injerencia.

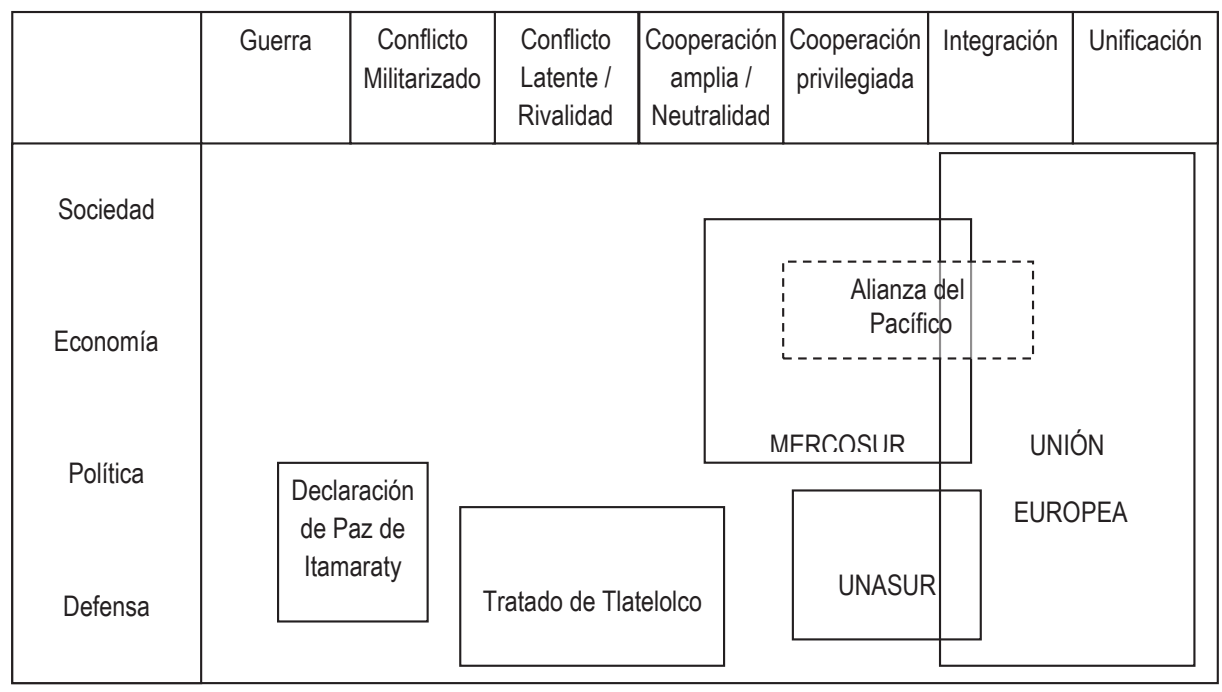

Fuente: Elaboración propia a partir de Briones y Álvarez, 2008: 23-31.

Con todo, es necesario poner en perspectiva el eventual agotamiento del «regionalismo abierto» y su reemplazo por una nueva agenda. Ejemplo de ello es el modelo de «regionalismo post liberal». Según Van Klaveren, a diferencia de iniciativas precedentes, actualmente el regionalismo adoptaría diferentes formas y estrategias, como serían las instancias bilaterales, multilaterales, regionales y subregionales. Todas ellas, además, con diferentes grados de institucionalización. Al mismo tiempo, el componente comercial característico del «regionalismo abierto» de los años noventa parece haber girado hacia proyectos asociados a temas de interés común para los países de la región, tales como recursos naturales, energía, tecnología, infraestructura, etc. Eso permitiría hablar de un "regionalismo heterodoxo» (Van Klaveren, 2012: 101).
Esta heterogeneidad regional se manifiesta también en las distintas opciones de vinculación con Estados Unidos adoptadas por los países de América Latina (Rusell y Tokatlian, 2009). La literatura ha reconocido la naturaleza ecléctica del regionalismo en América Latina (Oyarzún y Rojas, 2013) y la «fragmentación subregional» de los procesos de integración (Briceño, 2013). En esa perspectiva, mientras los países agrupados en la Alianza del Pacífico promueven una estrategia apegada a los principios del «regionalismo abierto ", léase principalmente apertura comercial, el perfil atlántico en materia de integración promovería el restablecimiento de cierto proteccionismo y un desacople respecto de Estados Unidos (Oyarzún y Rojas, 2013: 25). Así pues, los países que adhieren a la estrategia de suscribir tratados de libre comercio y 
los que conforman la Alianza del Pacífico representan un polo de «integración abierta». Por su parte, el «eje revisionista» explicaría el giro político que ha tomado el MERCOSUR. Finalmente, el «eje anti-sistémico» tiene su núcleo en la Alianza Bolivariana de Naciones (ALBA) (Briceño, 2013: 35). Mientras tanto, el eje del Pacífico se constituiría en «bastión aperturista» en una región cruzada por visiones críticas o de rechazo absoluto a la apertura global (Briceño, 2010: 58).

\section{CHILE: DE LA REINSERCIÓN AL REGIONALISMO ABIERTO}

En el contexto regional antes descrito, la política exterior chilena se ha desplegado a partir de dos procesos: el restablecimiento de la democracia y un nuevo esquema o estrategia de desarrollo cuya implementación comenzó bajo el autoritarismo de Pinochet. Asociada a lo primero, se inscribe la «reinserción internacional». En lo segundo, se encuentra la aplicación de una estrategia de negociaciones comerciales en línea con la política de apertura comercial (Van Klaveren, 2011; Wilhelmy y Durán, 2003; FLACSO, 2004). A estas vertientes de desarrollo principales se añadió la creciente participación de Chile en instancias multilaterales y de cooperación en materias de interés de la comunidad internacional, como la seguridad (Fuentes, 2007: 99-102).

El paraguas conceptual de la política exterior chilena en los años noventa habría sido el «regionalismo abierto». Partiendo de la base de que Chile no renunciaría a la orientación global de su política comercial (Van Klaveren, 2011), el «regionalismo abierto» permitió superar la ausencia de consensos entre los países de la región respecto de los modelos de desarrollo, pero también en lo relativo a otras materias políticas sensibles, como derechos humanos, seguridad internacional y medio ambiente (Van Klaveren, 1998: 77). Con todo, la estrategia de la política exterior chilena se habría basado en la libertad y la promoción del comercio global (Van Klaveren, 1998 y 2011).

De acuerdo con lo anterior, la política internacional chilena se ha caracterizado por su pragmatismo y por el énfasis en el aspecto económico-comercial. Todo ello a expensas del componente político de la política exterior e incluso de dar mayor profundidad a las relaciones vecinales (Oyarzún, 2013). En ese contexto general, en 2010 se produjo un vuelco en el proceso político: por primera vez en dos décadas desde la recuperación de la democracia, la oposición de partidos de derecha llegó a La Moneda, liderada por el candidato Sebastián Piñera Echeñique. Dado lo anterior, este artículo pasa revista a los principales elementos que caracterizan al gobierno de Piñera en su actuación internacional. Al respecto, un primer elemento que cabe revisar es la situación de los procesos de integración regional, habida cuenta de que en su gobierno (2010-2014) tiene lugar el ingreso de Chile a una nueva instancia 
compuesta por países latinoamericanos con salida al océano Pacífico. Esa es probablemente la innovación más relevante de la administración piñerista en lo que se refiere a la agenda internacional. Al mismo tiempo, como se examina a continuación, en otros aspectos como política comercial, relaciones vecinales y seguridad internacional, la continuidad respecto de lo realizado por las administraciones precedentes parece ser un rasgo dominante de la política exterior chilena.

\section{LA POLÍTICA EXTERIOR DE Piñera: AGENDA E Hitos}

\section{Ámbito programático}

Durante alrededor de dos años, el entonces candidato a la Presidencia de la República, Sebastián Piñera, se abocó a la tarea de elaborar un programa de gobierno de cara a los comicios presidenciales de 2009. En dicha competencia, Piñera se presentó como el candidato único de la Alianza por Chile. A continuación se muestran algunos de los aspectos más importantes del programa del abanderado derechista en lo que respecta a la política internacional. Ello es importante, toda vez que con dicho manifiesto Piñera llegó a La Moneda en 2010.

El acápite de política internacional de Piñera establecía siete ejes de lo que sería su gestión en dicho campo de la actividad estatal. Un primer eje corres- pondía al despliegue de una «verdadera Política Exterior de Estado». Eso consideraba responder a intereses tales como: preservación de la soberanía, autodeterminación e inserción global. Lo anterior, teniendo como objetivo general la continuidad de la política exterior del país, pero con una impronta de mayor profesionalismo y «acentuando el sentido de excelencia» (Piñera y Coalición por el Cambio, 2009: 116).

El segundo pilar de la política internacional contenida en el programa de Piñera decía relación con el fortalecimiento y resguardo de la normativa jurídica internacional. Como lo declaraba el manifiesto, Piñera se comprometía a fortalecer los principios de la política exterior seguida por Chile desde el retorno a la democracia, tales como:

- respeto del derecho internacional,

- intangibilidad de los tratados,

- respeto y promoción de la democracia y los derechos humanos,

- solución pacífica de las controversias,

- no intervención en los asuntos internos de los Estados,

- respeto al principio de igualdad de los Estados,

- libre determinación de los pueblos.

En tercer lugar, el manifiesto proponía como eje de acción en política exterior dar prioridad a la relación de Chile con la región y las naciones vecinas. A pesar de que se reconoce que Chile cuenta con una estrategia económica de orientación global y que, en consecuen- 
cia, sus intereses comerciales no necesariamente se circunscriben a América Latina, se proponía avanzar en: «(...) generar acuerdos estratégicos y una mayor integración, sin que ello implique una renuncia a nuestro exitoso modelo de desarrollo económico y social» (p. 117). De tal modo, se planteaba mejorar las relaciones vecinales dados los factores de unión que existirían entre Chile y los países de la región: lengua, territorio, cultura, historia, entre otros. Ello se reconoce como particularmente atingente en el caso de las naciones con las cuales se comparte frontera: Argentina, Bolivia y Perú.

Tal como se proponía que América Latina fuese una prioridad para la política internacional, el programa identificaba como cuarto eje de esa política avanzar en las relaciones con Estados Unidos y la Comunidad Europea, basándose en dos elementos. Primero, que Chile tiene intercambios comerciales privilegiados con ambos3 y segundo, el programa argumenta que, además de la dimensión económica, Chile compartiría con ellos un conjunto de valores, tales como el respeto de los derechos humanos, el Estado de derecho, el sistema político democrático y la libertad comercial.

Respecto de la región Asia-Pacífico, se reconoce su creciente dinamismo e

De acuerdo con los datos de 2013, después de China, Estados Unidos recibe el $12.6 \%$ de todas las exportaciones chilenas. Mientras tanto, la Unión Europea es el destino de más del $14 \%$ de las exportaciones nacionales (Ver cuadro 2). importancia, no obstante solo se menciona la necesidad de que Chile: «(...) siga incrementando sus relaciones con los países del Asia-Pacifico, considerando las potencialidades que presenta esta área dentro del concierto mundial» (p. 119).

La apertura comercial constituía un quinto eje de la política internacional delineada en el manifiesto. En ese sentido, planteaba la profundización del proceso de apertura comercial. El texto proponía mantener la orientación y principales elementos de dicha apertura en los ámbitos bilateral y multilateral, de acuerdo con lo obrado por las administraciones anteriores. Como se examina luego, lo anterior se tradujo en una fuerte línea de continuidad en los ámbitos señalados, particularmente en lo que refiere a los planos comercial y multilateral. También se destacaba la necesidad de fomentar la colaboración público-privada, así como la consolidación general de los instrumentos comerciales suscritos que son parte de la apertura exterior del país.

En sexto lugar, como eje de la política exterior el programa de gobierno de S. Piñera abordaba la defensa de la soberanía y el territorio. Aquí se incluían los derechos e intereses marítimos del país y también los intereses de Chile en la Antártica. En tal sentido, el programa declaraba que un principio esencial de la política exterior internacional correspondería a la proyección en el océano Pacífico y el mantenimiento de los intereses antárticos. En ese orden de ideas, el programa relevaba la nece- 
sidad de perfilar un nuevo rol de Chile en la cuenca del Pacífico. Ello, bajo el diagnóstico señalado en el programa de que la región: «(...) se distingue por su diversidad política, dinamismo económico y comercial, y por su proyección geoestratégica» (p. 123).

Un séptimo eje se relaciona con reformas administrativas que se consideraban necesarias para la gestión de la política exterior. En ese sentido, se propuso la modernización del Ministerio de Relaciones Exteriores (MINREL) en diferentes aspectos, tales como personal; evaluación de desempeño; profesionalismo.

Hasta aquí en lo que refiere a lo declarado por el entonces candidato Sebastián Piñera como sus compromisos en política exterior. A continuación se abordan los principales hechos y datos correspondientes a la gestión de Piñera en los ámbitos comercial, participación regional y multilateral, y en operaciones de paz. Asimismo, se recogen sumariamente algunos hechos relativos a las relaciones con naciones vecinas, en particular al diferendo de Chile y Perú zanjado por la Corte de la Haya en ese periodo presidencial.

\section{Ámbito comercial}

Pese a la ambiciosa agenda planteada en el programa, aquí se argumenta que la política internacional del gobierno de Sebastián Piñera estuvo marcada mayormente por hitos de continuidad que de cambio. En ese sentido, podemos resumir los hitos y hechos más importantes en cuatro ámbitos: comercial, regional, vecinal y seguridad internacional.

Aquí, se nota una continuidad de la política comercial abierta seguida por el país. Junto con mantener los tratados de comercio suscritos por los gobiernos de la Concertación, se persiguió celebrar otros o ampliar algunos. En este sentido, el hecho de sumar tratados o áreas nuevas a las existentes difícilmente puede concebirse como una ruptura con la política preexistente. Más aún, cuando habiendo celebrado tratados de libre comercio con los principales bloques económicos del mundo, las reducciones tarifarias que pueden perseguirse son relativamente marginales comparadas con lo que significaron los acuerdos con Estados Unidos, la Unión Europea, Japón y China.

\section{Un listado breve de hechos en el ámbito comercial puede ser el siguiente:}

El hito comercial más relevante posiblemente sea el acuerdo marco de la Alianza del Pacífico y su protocolo adicional, incorporado en la sección sobre el ámbito regional.

Otro evento trascendente fue la firma en Honolulu, Hawái, del Tratado de Libre Comercio Chile-Vietnam, el 11 de Noviembre de 2011, y que entró en vigor el 2 de diciembre de 2013 (Ministerio de RREE de Chile, 2014).

Finalmente cabe destacar el Acuerdo de Libre Comercio entre Chile y Turquía. Si bien este se había firmado en 
2009, durante la administración de Michelle Bachelet, recién vino a entrar en vigencia poco más de un año después, el 13 de enero de 2011 (Ministerio de RREE de Chile, 2011), ampliándolo al área de servicios. ${ }^{4}$

Los datos del comercio exterior chileno en los años 2011, 2012 y 2013 reflejan los principales intercambios de Chile en esta materia. La composición de las exportaciones nacionales da cuenta de la alta concentración en torno a la minería. El volumen total de exportaciones mineras alcanzó, en 2013, un monto total de 44.294 millones de dólares (ver el cuadro $\mathrm{N}^{\circ} 1$ ). El cobre representó casi la totalidad de las exportaciones mineras. Mientras tanto, en el mismo año, las exportaciones nacionales no correspondientes a la minería (frutas, vino, alimentos procesados, entre otras), totalizaron una cifra correspondiente a 30.551 millones de dólares. El volumen general del intercambio comercial se mantuvo estable los tres años considerados, situándose en una cifra promedio de $156 \mathrm{mil} \mathrm{mi-}$ llones de dólares.

En 2013, Chile alcanzó un superávit comercial de 2.377 millones de dólares, cifra inferior a la correspondiente a 2012 y marcadamente inferior a la balanza comercial de 2011. Lo ante-

4 «Chile y Turquía acuerdan ampliar el TLC al comercio de Servicios", Emol, 28 de marzo de 2013. Recuperado el 3 de junio de 2014 de: http://www.emol.com/ noticias/economia/2013/03/28/590724/ chile-y-turquia-acuerdan-ampiar-el-tlc-alsector-de-servicios.html rior, producto de un aumento en las importaciones y una reducción en las exportaciones nacionales (DIRECON, 2013).

A pesar de ser meramente descriptivos, estos datos reflejan una realidad más profunda: el modelo económico chileno se sigue sustentando, en primer lugar, por las exportaciones de cobre y en segundo lugar, por un número acotado de exportaciones vinculadas a materias primas. En ese sentido, la estructura productiva del país dista mucho de acercarse a la matriz económica de un país desarrollado (Kynge, 2014). Esto es congruente con el estudio del National Bureau of Economic Research que ubicó a la economía chilena como «mercado emergente frágil» (Aizenman, Binici y Hutchison, 2014). 
Cuadro $N^{\circ} 1$ : Comercio exterior chileno 2011-2013 (MM US\$).

\begin{tabular}{|c|c|c|c|}
\hline & 2011 & 2012 & 2013 \\
\hline Intercambio comercial & 156.319 & 157.745 & 156.989 \\
\hline Exportaciones total & 81.411 & 78.277 & 77.367 \\
\hline Total exportaciones mineras & 48.865 & 46.537 & 44.294 \\
\hline Exportaciones cobre total & 44.438 & 42.184 & 40.509 \\
\hline Resto minería exportaciones & 4.427 & 4.353 & 3.785 \\
\hline Exportaciones celulosa total & 2.600 & 2.303 & 2.522 \\
\hline Exportaciones no mineras ni celulosa total & 29.946 & 29.437 & 30.551 \\
\hline Frutas & 4.274 & 4.294 & 4.893 \\
\hline Alimentos procesados sin salmón & 5.793 & 5.614 & 5.528 \\
\hline Salmón & 1.853 & 1.980 & 2.760 \\
\hline Vino embotellado & 1.445 & 1.455 & 1.481 \\
\hline Forestal y muebles de la madera & 2.222 & 2.129 & 2.264 \\
\hline Químicos & 6.105 & 6.040 & 5.469 \\
\hline Productos metálicos, maquinaria, equipos & 2.916 & 2.872 & 3.035 \\
\hline Importaciones total (CIF) & 74.908 & 79.468 & 79.621 \\
\hline Bienes intermedios & 41.873 & 43.442 & 42.512 \\
\hline Petróleo & 6.498 & 6.108 & 6.633 \\
\hline Bienes de consumo & 19.847 & 19.859 & 21.658 \\
\hline Bienes de capital & 13.189 & 16.167 & 15.451 \\
\hline Saldo balanza comercial (FOB) & 10.792 & 3.422 & 2.377 \\
\hline
\end{tabular}

Fuente: DIRECON (2011, 2012 y 2013).

Como se muestra en el cuadro $\mathrm{N}^{\circ} 2$, el destino de las exportaciones chilenas en el periodo 2011-2013 tuvo a China como principal mercado, creciendo de una participación del $22,8 \%$ en 2011 a un $24,8 \%$ en 2013. Como socio comercial, la Unión Europea redujo su participación como plaza de las exportaciones chilenas: en 2011, el mercado europeo representó un $17,7 \%$, mientras que en 2012 se redujo a un $14,6 \%$. Estados Unidos mantuvo un promedio estable en el volumen de salida del comercio chileno. En 2011 las exportaciones a dicho país sumaron 9.047 millones de dólares. En 2013, la cifra ascendió a 9.756 millones de dólares. La impor- tancia del mercado asiático para las exportaciones de Chile también se refleja en el volumen del intercambio con Japón y Corea del Sur (un promedio de $10,5 \%$ y $5,5 \%$ de participación en el total de exportaciones nacionales, respectivamente). Sumados China, Japón y Corea del Sur, alcanzaron un 40,2\% del total de exportaciones nacionales en 2013. La cifra anterior contrasta con la situación del intercambio comercial a nivel regional. El MERCOSUR solo concentra un promedio de $7,9 \%$ de las exportaciones de Chile entre 2011 y 2013. La Comunidad Andina, por su parte, es destino del 6,2\% de dicho intercambio en el mismo periodo. Su- 
madas ambas instancias de integración， total de importaciones alcanzó 11.771 alcanzan un $14,1 \%$ del total de las millones de dólares $(15,7 \%)$, cayendo exportaciones nacionales.

a 9.823 millones de dólares en 2013

Cuadro N²: Exportaciones de Chile según acuerdo comercial (MMUS\$).

\begin{tabular}{|l|c|r|r|r|r|r|}
\hline \multirow{2}{*}{ Acuerdo Comercial } & \multicolumn{2}{|c|}{2011} & \multicolumn{2}{c|}{2012} & \multicolumn{2}{c|}{2013} \\
\hline China & 18.601 & $22,8 \%$ & 18.218 & $23,3 \%$ & 19.219 & $24,8 \%$ \\
\hline Estados Unidos & 9.047 & $11,1 \%$ & 9.580 & $12,2 \%$ & 9.756 & $12,6 \%$ \\
\hline Unión Europea & 14.391 & $17,7 \%$ & 11.970 & $15,3 \%$ & 11.278 & $14,6 \%$ \\
\hline Japón & 9.009 & $11,1 \%$ & 8.384 & $10,7 \%$ & 7.661 & $9,9 \%$ \\
\hline MERCOSUR & 6.456 & $7,9 \%$ & 6.053 & $7,7 \%$ & 6.160 & $8,0 \%$ \\
\hline Comunidad Andina & 4.989 & $6,1 \%$ & 4.798 & $6,1 \%$ & 5.059 & $6,5 \%$ \\
\hline Corea del Sur & 4.448 & $5,5 \%$ & 4.551 & $5,8 \%$ & 4.272 & $5,5 \%$ \\
\hline India & 1.913 & $2,4 \%$ & 2.586 & $3,3 \%$ & 2.304 & $3,0 \%$ \\
\hline Canadá & 1.476 & $1,8 \%$ & 1.283 & $1,6 \%$ & 1.418 & $1,8 \%$ \\
\hline México & 1.827 & $2,2 \%$ & 1.346 & $1,7 \%$ & 1.321 & $1,7 \%$ \\
\hline Australia & 925 & $1,1 \%$ & 1.250 & $1,6 \%$ & 801 & $1,0 \%$ \\
\hline Venezuela & 685 & $0,8 \%$ & 692 & $0,9 \%$ & 522 & $0,7 \%$ \\
\hline Turquía & 456 & $0,6 \%$ & 462 & $0,6 \%$ & 366 & $0,5 \%$ \\
\hline Vietnam & 334 & $0,4 \%$ & 372 & $0,5 \%$ & 307 & $0,4 \%$ \\
\hline Resto & 6.852 & $8,4 \%$ & 6.731 & $8,6 \%$ & 6.922 & $8,9 \%$ \\
\hline Total exportaciones & 81.411 & $100 \%$ & 78.277 & $100 \%$ & 77.367 & $100 \%$ \\
\hline
\end{tabular}

Fuente: DIRECON (2011, 2012 y 2013).

En lo que respecta a las importaciones en el periodo analizado, Estados Unidos sigue siendo la fuente principal. En 2013, el total importado desde dicho país se elevó a 16.088 millones de dólares, representando un 20,2\% de las importaciones chilenas. China ha aumentado su participación en el total de bienes, servicios y capital importado por Chile. En 2011, Chile importó un total de 12.696 millones de dólares (16,9\%). En 2013 ese guarismo aumentó, alcanzando China una participación de $19,7 \%$ en el monto total de importaciones nacionales, que fue de 15.702 millones de dólares. En cuanto al MERCOSUR, en 2011 el volumen
(12,3\% de participación). Los bienes, servicios y capital importado de la Comunidad Andina se mantuvo estable durante los tres años considerados (ver el cuadro $\mathrm{N}^{\circ} 3$ ). Esto refleja que, si bien de manera lenta, las potencias económicas desarrolladas (Estados Unidos, la Unión Europea y Japón) han perdido relevancia relativa, a la vez ha aumentado la importancia de China, no solo como principal socio comercial, sino como uno cuyo peso sigue creciendo. 
Cuadro N³: Importaciones realizadas por Chile según acuerdo comercial (MMUS\$).

\begin{tabular}{|l|l|l|l|l|l|l|}
\hline \multirow{2}{*}{ Acuerdo comercial } & \multicolumn{2}{|c|}{2011} & \multicolumn{2}{c|}{2012} & \multicolumn{2}{c|}{2013} \\
\hline China & 12.696 & $16,9 \%$ & 14.432 & $18,2 \%$ & 15.702 & $19,7 \%$ \\
\hline Estados Unidos & 15.092 & $20,1 \%$ & 18.188 & $22,9 \%$ & 16.088 & $20,2 \%$ \\
\hline Unión Europea & 10.350 & $13,8 \%$ & 10.635 & $13,4 \%$ & 13.187 & $16,6 \%$ \\
\hline Japón & 2.958 & $3,9 \%$ & 2.596 & $3,3 \%$ & 2.495 & $3,1 \%$ \\
\hline MERCOSUR & 11.771 & $15,7 \%$ & 10.952 & $13,8 \%$ & 9.823 & $12,3 \%$ \\
\hline Comunidad Andina & 5.693 & $7,6 \%$ & 6.650 & $8,4 \%$ & 6.135 & $7,7 \%$ \\
\hline Corea del Sur & 2.730 & $3,6 \%$ & 2.604 & $3,3 \%$ & 2.771 & $3,5 \%$ \\
\hline India & 500 & $0,7 \%$ & 711 & $0,9 \%$ & 739 & $0,9 \%$ \\
\hline Canadá & 913 & $1,2 \%$ & 1.032 & $1,3 \%$ & 1.544 & $1,9 \%$ \\
\hline México & 2.527 & $3,4 \%$ & 2.608 & $3,3 \$$ & 2.543 & $3,2 \%$ \\
\hline Australia & 521 & $0,7 \%$ & 474 & $0,6 \%$ & 414 & $0,5 \%$ \\
\hline Venezuela & 185 & $0,2 \%$ & 197 & $0,2 \%$ & 133 & $0,2 \%$ \\
\hline Turquía & 168 & $0,2 \%$ & 217 & $0,3 \%$ & 264 & $0,3 \%$ \\
\hline Resto & 8.806 & $11,8 \%$ & 8.171 & $10,3 \%$ & 7.783 & $9,8 \%$ \\
\hline Total exportaciones & 74.908 & $100 \%$ & 79.468 & $100 \%$ & 79.621 & $100 \%$ \\
\hline
\end{tabular}

Fuente: DIRECON (2011, 2012 y 2013).

\section{Ámbito regional}

Aquí es importante destacar la existencia de eventos en tres organismos regionales: la CELAC, UNASUR y la Alianza del Pacífico. Respecto de los dos primeros, si bien hubo varios sucesos de importancia, el gobierno de Piñera pareció asumir más un rol de continuador que de protagonista. Así, a diferencia de su papel en la Alianza del Pacífico, si bien no se opuso ni abandonó la Comunidad de Estados Latinoamericanos y del Caribe (CELAC) ni la UNASUR, mantuvo una posición bastante pasiva.

Dentro de la CELAC, cabe destacar dos eventos:
- Presidencia pro-tempore de la Comunidad, del 3 de diciembre de 2011 al 28 de enero de 2013.

- Realización de la Primera cumbre CELAC-UE en Santiago de Chile, el 26 de enero de 2013.

En cuanto a la UNASUR, durante el gobierno de Sebastián Piñera se crearon las siguientes instancias:

- Consejo Energético Suramericano, 4 de mayo de 2010 en la ciudad Los Cardales, Argentina.

- Secretaría UNASUR-Haití, comenzó sus actividades el 31 de agosto de 2010.

- El Consejo Suramericano de Economía y Finanzas, en Georgetown, Guyana, el 26 de noviembre de 2010. 
- Centro de Estudios Estratégicos de Defensa (CEED), inaugurado en Buenos Aires el 27 de mayo de 2011.

- Instituto Suramericano de Gobierno en Salud (ISAGS), en Río de Janeiro desde el 25 de julio de 2011.

- Consejo Electoral de UNASUR, el 29 de octubre de 2011.

- Consejo Suramericano de Educación, el 30 de noviembre de 2012 en Lima, Perú.

- Consejo Suramericano de Cultura, el 30 de noviembre de 2012 en Lima, Perú.

- Consejo Suramericano de Ciencia, Tecnología e Innovación, el 30 de noviembre de 2012 en Lima, Perú.

- Consejo Suramericano en materia de Seguridad Ciudadana, Justicia y Coordinación de Acciones contra la Delincuencia Organizada Trasnacional, el 30 de noviembre de 2012 en Lima, Perú.

- Grupo de Trabajo de Solución de Controversias en Inversiones.

En este sentido es importante hacer una precisión. La existencia de los Consejos antes señalados es anterior a la fecha indicada, pues esta refleja solamente su instalación como órgano oficial y el establecimiento de una sede permanente para los mismos (UNASUR, 2011).

El denominado "eje del Pacífico", que pasó pronto a convertirse en la «Alianza del Pacífico", es el gran hito de política internacional del gobierno de Sebastián Piñera. Chile es miembro pleno de esta instancia, junto a Colom- bia, México y Perú5. La razón para afirmar que es un hito distintivo es que ocurrió en un solo periodo de gobierno, mediante una agenda intensa en reuniones que comenzó con la mera cercanía de ideas de cuatro presidentes (además del chileno, Alan García, Juan Manuel Santos y Felipe Calderón). Esta agenda, sin embargo, concluyó, en apenas un par de años, la redacción y firma de un acuerdo amplio de intercambio de bienes, servicios y circulación de personas.

Dentro de la Alianza del Pacífico se desarrollaron ocho cumbres de presidentes:

- Declaración Presidencial sobre la Alianza del Pacífico. Lima, 28 de abril de 2011.

- Declaración de Mérida de la II Cumbre. Mérida, Yucatán, México, 4 de diciembre de 2011.

- III Cumbre de la Alianza del Pacífico se realiza en forma virtual.

- Declaración de la IV Cumbre de la Alianza del Pacífico. Paranal, Antofagasta, Chile, 6 de junio de 2012.

- Declaración conjunta de Presidentes de la Alianza del Pacífico. V Cumbre de la Alianza del Pacífico - XXII Cumbre Iberoamericana.

- VI Cumbre de Presidentes de la Alianza del Pacífico. Santiago de Chile, 26 de enero de 2013 -I Cumbre CELAC-UE.

En esta iniciativa no participan otros países de la región como Argentina, Brasil, Ecuador o Venezuela. 
- Declaración de Cali, VII Cumbre de la Alianza del Pacífico. Cali, Colombia, 23 de mayo de 2013.

- Declaración de la VIII Cumbre de la Alianza del Pacífico. Cartagena de Indias, Colombia, 10 de febrero de 2014.

- Acuerdo Marco de la Alianza del Pacífico de 2012 y su Protocolo Adicional de 2014.

En este contexto el acuerdo marco es el documento más importante del organismo por dos razones. Primero, detalla el contenido de los objetivos, requisitos y metas de la Alianza. Segundo, se diferencia de las declaraciones por su contenido vinculante. Dentro de ese contenido destacan:

Los requisitos para ser miembro de la Alianza: vigencia del Estado de derecho, la democracia y las constituciones; separación de los poderes del Estado; y respeto de los derechos humanos (Alianza del Pacífico, 2012c).

Los objetivos: creación de un área de libre circulación de bienes, servicios, capitales y personas; impulso al crecimiento como mecanismo de superación de la desigualdad; y transformación en una plataforma de articulación al mundo (Alianza del Pacífico, 2012c).

Como lo declara en su documento constitutivo, la Alianza se presenta como una instancia de integración regional entre naciones que defienden el libre comercio y la apertura económica. En ese sentido, busca fomentar el «regionalismo abierto", que sirva de plataforma para que los países miem- bros se inserten en las redes económicas globales (Alianza del Pacífico, 2012c).

El dinamismo económico que caracteriza a los países integrantes de la Alianza del Pacífico no ha pasado inadvertido. A diferencia de otros proyectos de integración en la región carentes de capacidad económica basal (Burges, 2005), las economías de los países miembros concentran alrededor del 35 por ciento del producto interno bruto regional. La sostenida tendencia de crecimiento en el producto en países como Chile, Colombia y Perú, daría sustento a la novel instancia de integración (Forster, 2013).

Sin embargo, la formación de este nuevo bloque no ha sido bien recibida por todos los actores. A nivel regional, el ex presidente brasileño Lula da Silva ha planteado reparos a la iniciativa, acusándola de actuar contra corriente de la integración en la región6, o bien siendo catalogada como excluyente por actores claves de la diplomacia brasileña.7 Por su parte, el mandatario ecuatoriano Rafael Correa cuestionó el perfil «neoliberal» que tendría la Alianza del Pacífico.8 Con todo, es notable que las diferencias ideológicas con Venezuela no hayan decantado en una ruptura mayor de las relaciones de Chile con ese país, que podrían haberse

6 «La Alianza del Pacífico logra consolidarse a menos de dos años de su creación», $L a$ Tercera, 16 de febrero de 2014.

7 «Marco Aurelio García: La Alianza del Pacífico no es relevante como se plantea», El Mercurio, 16 de marzo de 2014.

8 «Titanes del Pacífico», Revista Qué Pasa, 23 de octubre de 2013. 
tornado mucho menos amistosas de lo que se mantuvieron.

\section{Ámbito relaciones vecinales}

Dentro de las relaciones vecinales, no hubo grandes hitos. Por el contrario, la diferencia ideológica con los presidentes de Bolivia y Argentina parece haber llevado a un enfriamiento de las relaciones que no empeoró las condiciones, pero tampoco marcó avances. Distinto es el caso de Perú. Si en el sentido económico Chile y Perú estaban dentro de la Alianza del Pacífico, en el sentido territorial, tuvo lugar el contencioso en la Corte Internacional de Justicia de la Haya.

Los hechos más relevantes del proceso ante la Corte Internacional de la Haya acaecidos durante el gobierno de Sebastián Piñera son los siguientes:

- Presentación de la réplica peruana. 9 de noviembre de 2010 (ICJ, 2010).

- Presentación de la dúplica chilena. 11 de julio de 2011 (ICJ, 2011).

- Audiencias públicas. 3, 4, 6, 7, 11 y 14 de diciembre de 2012 (ICJ, 2012).

- Sentenciade la Corte. 27 de enero de 2014 (ICJ, 2014).

Ahora, dentro del contexto adverso que significó el juicio, los resultados fueron valorados como una pérdida moderada por parte de Chile. El propio Presidente sostuvo que el fallo «ha confirmado en lo sustancial los argumentos de la posición chilena».9 Aun cuando el fallo distaba mucho de la posición oficial y pública de Chile, se percibió como beneficioso el cerrar un capítulo tenso de la relación bilateral, que mantuvo en ascuas el diálogo entre ambas naciones. Esto porque si bien, como señaló el entonces canciller peruano, una demanda ante La Haya «no es un acto inamistoso»10, tampoco es precisamente un gesto amistoso. Terminar ese proceso permitió concluir un período en el que las declaraciones públicas al respecto eran cuidadosamente vigiladas, no tanto por el impacto que podrían tener ante la Corte, sino por el peso sobre la opinión pública de ambos países.

\section{Ámbito de seguridad internacional}

En este ámbito la política exterior de Chile no tuvo grandes giros. La participación del presidente Piñera en la Cumbre de Seguridad Nuclear, el 2010 en Washington (Farzaneh, Swift y Williams, 2010) y el 2012 en Seúl, lo hizo figurar entre los líderes mundiales, pero cabe preguntarse si su participación tuvo impacto en los asuntos tratados

9 «Piñera afirma que el fallo de La Haya ha confirmado los argumentos de Chile», Terra, 27 de enero de 2014. Recuperado el 16 de junio de 2014 de: http://noticias. terra.cl/

10 "Canciller aseguró que demanda marítima a Chile no es acto inamistoso", $E l$ Comercio, 15 de junio de 2012. Recuperado el 15 de junio de 2014 de: http://elcomercio.pe/ 
en la cumbre, o si su mayor beneficio fue una oportunidad para encuentros bilaterales que se efectuaron en forma paralela.

Por otra parte, en materia de defensa la diplomacia cumplió su papel activo tradicional, en el que cabe mencionar la participación del ministro de Defensa Andrés Allamand en el ejercicio Rimpac 2012.11 Al mismo tiempo, Chile tuvo un activo rol en la aprobación del «Sistema de Colaboración Internacional para el Caso de Desastres Naturales», en la X Conferencia de Ministros de las Américas realizada en Uruguay en 2012. Dicha iniciativa buscaba que las capacidades militares de los países de la región fuesen utilizadas en tareas de cooperación frente a catástrofes naturales.12 Otro hecho relevante fue que un general chileno asumió el 2013 la presidencia de la Junta Interamericana de Defensa en Washington DC.13

11 «En Hawaii: Ministro Allamand y comandante en jefe de la Armada participan en ejercicio Rimpac 2012», La Segunda online, 21 de julio de 2012. Recuperado el 21 de agosto de 2014 de: http://www.lasegunda.com

12 «Ministro de Defensa anuncia creación de sistema de colaboración para afrontar catástrofes naturales", Ministerio de Defensa, 10 de octubre de 2012. Recuperado el 23 de agosto de 2014. Disponible en: http://www.defensa.cl/ noticias/ministro-de-defensa-anunciacreacion-de-sistema-de-colaboracionpara-afrontar-catastrofes-naturales/

13 "General chileno asume Presidencia de la Junta Interamericana de Defensa», Subsecretaría para las Fuerzas Armadas, 29 de junio de 2013. Recuperado el 21 de agosto de 2014 de: http://www.ssffaa.cl/
Chile también asumió uno de los asientos no permanentes correspondientes a América Latina en el Consejo de Seguridad de Naciones Unidas, del $1^{\circ}$ de enero de 2014 al 31 de diciembre de 2015. El período efectivo en que Chile fue miembro del Consejo, bajo el gobierno de Sebastián Piñera, fue sin embargo breve: del $1^{\circ}$ de enero de 2014 al 11 de marzo de ese mismo año. En ese período se aprobaron las resoluciones 2133 a 2143, todas ellas por unanimidad de sus miembros (ONU, 2014a):

- Resolución 2133, del 27 de enero de 2014, sobre amenazas a la paz y la seguridad internacionales causadas por actos terroristas.

- Resolución 2134, del 28 de enero de 2014, sobre la situación en la República Centroafricana.

- Resolución 2135, del 30 de enero de 2014, sobre la situación en Chipre.

- Resolución 2136, del 30 de enero de 2014, sobre la situación relativa a la República Democrática del Congo.

- Resolución 2137, del 13 de febrero de 2014, sobre la situación en Burundi.

- Resolución 2138, del 13 de febrero de 2014, sobre los Informes del Secretario General sobre Sudán.

- Resolución 2139, del 22 de febrero de 2014, sobre la situación en el Oriente Medio.

- Resolución 2140, del 26 de febrero de 2014, sobre la situación en el Oriente Medio.

- Resolución 2141, del 5 de marzo de 2014, sobre No proliferación/República Popular Democrática de Corea. 
- Resolución 2142, del 5 de marzo de 2014, sobre la situación en Somalia.

- Resolución 2143, del 7 de marzo de 2014, sobre los niños y los conflictos armados.

De las resoluciones anteriores la más relevante es la 2140, debido a que es resolutiva. Esto, dado que establece prohibiciones de viaje y sobre recursos a personas o grupos que puedan afectar la paz y la seguridad, en especial en vistas del proceso político en Yemen (ONU, 2014b).

La participación de Chile en las Operaciones de Paz de Naciones Unidas se mantuvo mayormente inalterada, tanto en las operaciones en las que Chile contribuyó (Estado Mayor Conjunto, 2014), como en el número de efectivos (ONU, 2014c) desplegados en ellas, salvo la lenta pero sostenida reducción de las fuerzas destacadas en Haití. Las operaciones activas de las Naciones Unidas
Unión Europea en Bosnia Herzegovina (EUFOR Althea), con alrededor de 15 efectivos del ejército (Estado Mayor Conjunto, 2014).

\section{CONSIDERACIONES FINALES}

Por tratarse de un simple racconto, este artículo no tiene pretensiones teóricas ni pone sobre la mesa mayores implicaciones analíticas. Simplemente, y a riesgo de no sopesar adecuadamente la evolución diacrónica, se intenta recoger la interrogante de si la alternancia en el gobierno, luego de dos décadas, trajo aparejada o no un cambio en la agenda de la política exterior en el caso chileno. Por una parte, se concluye, resultaba algo esperable que un gobierno de derecha tuviese visiones o plantease alianzas diferentes a las de la coalición de gobierno que lo antecedió. Pero por otro, la política exterior implementada en los gobiernos de la coalición

Cuadro $N^{\circ} 4$ : Número total de efectivos (militares en terreno, policías y expertos) a marzo del año seleccionado.

\begin{tabular}{|l|r|r|r|r|r|}
\hline Misión & \multicolumn{2}{|c|}{2010} & \multicolumn{2}{|c|}{2011} & \multicolumn{2}{|c|}{2012} & \multicolumn{2}{|c|}{2013} & \multicolumn{1}{|c|}{2014} \\
\hline Haití (MINUSTAH) & 518 & 515 & 509 & 477 & 425 \\
\hline Chipre (UNFICYP) & 15 & 15 & 15 & 14 & 14 \\
\hline India - Pakistán (UNMOGIP) & 2 & 2 & 2 & 2 & 2 \\
\hline Medio Oriente (UNTSO) & 3 & 3 & 3 & 3 & 3 \\
\hline
\end{tabular}

Fuente: ONU (2014c).

en que Chile estuvo involucrado y el número de efectivos están resumidos en el cuadro $\mathrm{N}^{\circ} 4$. A esas operaciones se suma la participación en la Misión de la de centro izquierda, mediante la firma de acuerdos de libre comercio con los principales países y bloques comerciales de los que el país es socio, resultaba 
congruente con los postulados de la derecha. En ese mismo orden de ideas, los bloques comerciales menos flexibles, como MERCOSUR, de los que Chile no es parte, no parecieron generar mayor interés en el nuevo gobierno, y tampoco había figurado en la agenda de las administraciones precedentes. De igual modo, la participación de Chile en otras instancias regionales o globales tampoco sufrió una alteración sustantiva.

A la luz de los datos revisados aquí, resulta plausible concluir que la política internacional del gobierno de Sebastián Piñera tuvo dos sellos claros. Uno de continuidad en los ámbitos de comercio, cooperación regional, multilateral y seguridad internacional. Este continuismo se hace inteligible a través de la suscripción de nuevos acuerdos de libre comercio, el mantenimiento de la participación en operaciones de paz, o la participación en foros regionales con regularidad. La mera preservación de muchos de los acuerdos suscritos y la permanencia en la UNASUR son muestra clara de una valoración positiva de las acciones internacionales de sus predecesores y la necesidad de conservarlas. El sello de innovación parcial fue respecto del regionalismo y la posición de Chile en las instancias de integración existentes en la región. Lo primero se aprecia tanto en los actos y decisiones de política exterior adoptados como también en el discurso reflejado en el programa de gobierno de Piñera.

Se aprecia un distintivo énfasis en reafirmar marcadamente el compromiso con el libre comercio, en la línea de lo que Briceño (2003: 15) llamó «eje de integración abierta», al participar en la formación de la Alianza del Pacífico. En este sentido, el gesto puede interpretarse como continuista o rupturista según dónde se sitúe la línea de base del análisis. Para la política exterior chilena de las últimas décadas, centrada en la promoción del comercio internacional mediante la firma de tratados de libre comercio, la Alianza del Pacífico es continuidad o profundización. Para una buena parte de la región, que a finales de los años noventa terminó considerando al «neoliberalismo y al consenso de Washington desacreditados» (Oelsner 2013: 121), puede considerarse más bien como rupturista o innovadora. Estas dos líneas de base para la comparación son las que permiten explicar la existencia de visiones contrapuestas sobre la alianza.

En el plano vecinal, las diferencias ideológicas con los gobiernos de Argentina y Bolivia parecen no haber provocado un mayor distanciamiento de las relaciones, particularmente con el primer país. Pero tampoco, como es reconocido ampliamente entre especialistas y expertos, se lograron avances significativos. En el caso de las relaciones con Perú, al gobierno de Piñera le correspondió continuar con la defensa jurídica del país frente a la demanda presentada por dicho país en 2008 en la Corte Internacional de Justicia. El fallo, emitido en el verano de 2014, dio término al contencioso, pero no definió claramente si ello conlleva la apertura de una nueva etapa en las relaciones. 
Paralelamente a dicho eje de conflicto, se desarrolló una agenda de cooperación, al ser Chile y Perú miembros de la Alianza del Pacífico, probablemente la acción internacional más importante de la administración de Piñera.

En ese sentido, la política internacional de Sebastián Piñera puede ser considerada en términos gruesos como de continuidad general. Ahora, si bien se trataría de una gran continuación de la política internacional chilena implementada por los gobiernos de la Concertación de Partidos por la Democracia, también se aprecia una toma de posición parcialmente innovadora frente a las alternativas de integración presentes en la región. Dicha innovación refiere al grado de compromiso adoptado por nuestro país en una instancia específica, pero no a sus objetivos generales ni lineamientos. Estos últimos, como se revisó en este artículo, son coincidentes con el esquema del «regionalismo abierto» implementado por Chile desde la reinauguración de la democracia en 1990. La continuidad en el tiempo de este último énfasis o faceta innovadora de la política exterior del gobierno de derecha, es algo que cabrá evaluar luego de la administración de Michelle Bachelet, que se inauguró en marzo de 2014.

\section{Bibliografía}

Alianza del Pacífico (2011a). Declaración Presidencial sobre la Alianza del Pacífico. Recuperado el 19 de mayo de 2014 de: http://alianzapacifico.net/
Alianza del Pacífico (2011b). Declaración de Mérida de la II Cumbre de la Alianza del Pacífico. Recuperado el 19 de mayo de 2014 de: http://alianzapacifico.net/

Alianza del Pacífico (2012a). Declaración de Paranal. Recuperado el 20 de mayo de 2014 de: http://alianzapacifico.net/

Alianza del Pacífico (2012b). Declaración Conjunta Presidencial de la Alianza del Pacífico. Recuperado el 20 de mayo de 2014 de: http://alianzapacifico.net/

Alianza del Pacífico (2012c). Acuerdo Marco. Recuperado el 22 de mayo de 2014 de: http://alianzapacifico.net/

Alianza del Pacífico (2013a). Declaración de Santiago. Recuperado el 23 de mayo de 2014 de: http://alianzapacifico.net/

Alianza del Pacífico (2013b). Declaración de Cali. Recuperado el 23 de mayo de 2014 de: http://alianzapacifico.net/

Alianza del Pacífico (2014a). Declaración de Cartagena. Recuperado el 23 de mayo de 2014 de: http://alianzapacifico.net/

Alianza del Pacífico (2014b). Protocolo Adicional del Acuerdo Marco. Recuperado el 19 de mayo de 2014 de: http://alianzapacifico.net/

Aizenman, Joshua; Binici, Mahir \& Hutchison, Michael (2014, marzo). «The Transmission of Federal Reserve Tapering News to Emerging Financial Markets». The National Bureau of Economic Research, Working Paper 19980. Recuperado el 15 de julio 2014 de: http://www.nber.org/ papers/w19980

Bernal-Meza, Raúl (2002). «A política exterior do Brasil: 1990-2002». Revista Brasileira de Política Internacional 45(1): 36-71.

Briceño, José (2010). «La iniciativa del Arco del Pacífico Latinoamericano. Un nuevo actor en el escenario de la integración regional». Nueva Sociedad 228: 44-60.

Briceño, José (2013). «Ejes y modelos en la etapa actual de la integración económica regional en América Latina». Estudios Internacionales 175: 9-39.

Briones, Sebastián \& Álvarez, Rodrigo (2008). ¿Construyendo confianzas? Fronteras, 
FFAA y Política en América Latina. Santiago, FLACSO-Chile.

Burges, Sean (2005). "Bounded by the reality of trade: practical limits to a South American region». Cambridge Review of International Affairs 18(3): 437-454.

Busso, Anabella (2014). «Los vaivenes de la política exterior argentina re-democratizada (1983-2013). Reflexiones sobre el impacto de los condicionantes internos». Estudios Internacionales 177: 9-33.

Caruson, Kiki \& Farrar-Myers, Victoria (2007). «Promoting the President's Foreign Policy Agenda: Presidential Use of Executive Agreements as Policy Vehicles». Political Research Quarterly 60(4): 631644.

CEPAL (1994). El regionalismo abierto en América Latina y el Caribe. La integración económica al servicio de la transformación productiva con equidad. Libros de la CEPAL N³9: Santiago, Chile.

Chodor, Tom \& McCarthy-Jones, Anthea (2013). "Post-Liberal Regionalism in Latin America and the Influence of Hugo Chávez». Journal of Iberian and Latin American Research 19(2): 211-223.

Costa, Alcides (1999). «Parcerias estratégicas no contexto da política exterior brasileira: implicações para o Mercosul». Revista Brasileira de Política Internacional 42(2): 52-80.

Forster, Magdalena (2013). «The Pacific Alliance - Latin America's new stars». Talking Point, Deutsche Bank Research, August 27. Disponible en: www.dbresearch.com

Estado Mayor Conjunto (2014). Operaciones de Paz. Recuperado de: http://www.emco. mil.cl/?page_id=111

Farzaneh, Kavyan; Swift, Andrew \& Williams, Peter (2010). "Guess Who's Coming to Dinner?». Foreign Policy, April 9. Recuperado el 21 de agosto 2014 de: http:// www.foreignpolicy.com/

Fermín, Eudis (2012). «Hugo Chávez: de la idea de Confederación de Estados a la conformación de la Alianza Bolivariana para los Pueblos de Nuestra América».
Universitas: Relações Internacionais 10(1): 53-68.

Fuentes, Claudio (2007). «Internacionalización sin «modernización». El caso del Ministerio de Relaciones Exteriores de Chile». América Latina Hoy 46: 97-117.

Kynge, James (2014). «EM held back by weak global recovery». Financial Times, March 23. Recuperado de: http://www.ft.com/ $\mathrm{intl} / \mathrm{cms} / \mathrm{s} / 0 / \mathrm{fc} 581 \mathrm{e} 50-\mathrm{b} 272-11 \mathrm{e} 3-$ b891-00144feabdc0.html\#slide0

Gardini, Gian Luca (2010). «Proyectos de integración regional sudamericana: hacia una teoría de convergencia regional». Relaciones Internacionales 15: 11-31.

Grugel, Jean \& Riggirozzi, Pía (2012). «Post-neoliberalism in Latin America: Rebuilding and Reclaiming the State after Crisis». Development and Change 43(1): 1-21.

Grugel, Jean; Riggirozzi, Pía \& Thirkell-White, Ben (2008). «Beyond the Washington Consensus? Asia and Latin America in search of more autonomous development». International Affairs 84(3): 499517.

International Court Of Justice, ICJ (2010). Reply of Peru. Maritime Dispute (Peru v. Chile). Recuperado de: http://www. icj-cij.org/

International Court Of Justice, ICJ (2011). Rejoinder of Chile. Maritime Dispute (Peru v. Chile). Recuperado de: http:// www.icj-cij.org/

International Court Of Justice, ICJ (2012). Oral Proceedings. Maritime Dispute (Peru v. Chile). Recuperado de: http://www. icj-cij.org/

International Court Of Justice, ICJ (2014). Judgments. Maritime Dispute (Peru v. Chile). Recuperado de: http://www.icj-cij.org/ Malamud, Andrés \& Gardini, Gian Luca (2012). «Has Regionalism Peaked? The Latin American Quagmire and its Lessons». The International Spectator: Italian Journal of International Affairs 47(1): 116-133.

Ministerio de Relaciones Exteriores, Chile (2011). Decreto Supremo N¹7, 13 de 
Enero de 2011. Diario Oficial, 01 de Marzo de 2011.

Ministerio de Relaciones Exteriores, Chile (2014). Decreto Supremo N¹39, 2 de diciembre de 2013. Diario Oficial, 04 de Febrero de 2014.

McAvoy, Gregory E. (2006). «Stability and change: The time varying impact of economic and foreign policy evaluations on presidential approval». Political Research Quarterly 59(1): 71-83.

MacKuen, Michael (1983). «Political Drama, Economic Conditions, and the Dynamics of Presidential Popularity». American Journal of Political Science 27(2): 165.

Marra, Robin; Ostrom, Charles \& Simon, Dennis (1990). «Foreign Policy and Presidential Popularity: Creating Windows of Opportunity in the Perpetual Election». The Journal of Conflict Resolution 34(4), December: 588-623.

Morandé, José (2003). «The Invisible Hand and Contemporary Foreign Policy». En: Mora, F. \& Hey, J. (Eds.). Latin American and Caribbean Foreign Policy. Rowmany Little Publishers, Inc.

Nolte, Detlef \& Wehner, Leslie (2013). «The Pacific Alliance Casts Its Clouds over Latin America». Focus 8. German Institute for Global Affairs (GIGA). Disponible en: www.giga-hamburg.de/giga-focus

Oelsner, Andrea (2013). «The Institutional Identity of Regional Organizations, Or Mercosur's Identity Crisis». International Studies Quarterly 57: 115-127.

ONU. Consejo de Seguridad (2014a). Resoluciones [. Recuperado el 15 de junio de 2014 de: http://www.un.org/es/sc/documents/resolutions/

ONU. Consejo de Seguridad (2014b). Resolución 2140. Recuperado el 15 de junio de 2014 de: http://www.un.org/es/sc/ documents/resolutions/

ONU. United Nations Peacekeeping (2014c). Troop and police contributors. Recuperado el 5 de junio de 2014 de: http:// www.un.org/en/peacekeeping/resources/ statistics/contributors.shtml
Onuki, Janina \& Oliveira, Amâncio Jorge de (2006). «Eleições, política externa e integração regional». Revista de Sociologia e Política 27 (novembro): 145-155.

Oyarzún, Lorena \& Rojas, Federico (2013). «La Alianza del Pacífico en América Latina. ¿Contrapeso regional?». Cuadernos sobre Relaciones Internacionales, Regionalismo y Desarrollo 8(16): 9-30.

Oyarzún, Lorena (2013). «When Trade Policy is Not Enough: Opportunities and Challenges for Chile's International Insertion». Journal of Iberian and Latin American Research 19(2): 268-285.

Phillips, Nicola (2003). "The rise and fall of open regionalism? Comparative reflections on regional governance in the Southern Cone of Latin America». Third World Quarterly 24 (2): 217-234.

Piñera, Sebastián \& Coalición por el Cambio (2009). «Programa de Gobierno para el cambio, el futuro y la esperanza (Chile 2010- 2014)». Accedido el 23 de agosto 2014 disponible en: http://www.umayor. cl/gestionpublica/descargables/docs/programa_gobierno_2010.pdf

Riggirozzi, Pía (2012). «Region, regionness and regionalism in Latin America: towards a new synthesis». New Political Economy 17(4): 421-443.

Riggirozzi, Pía \& Tussie, Diana (2012). «The Rise of Post-Hegemonic Regionalism in Latin America». En: Riggirozzi, P. \& Tussie, D. (Eds.), The Rise of Post-Hegemonic Regionalism. The Case of Latin America. Springer: 1-16.

Russell, Roberto \& Tokatlian, Juan (2009). «Modelos de política exterior y opciones estratégicas. El caso de América Latina frente a Estados Unidos». Revista CIDOB d'Afers Internacionals 85-86: 211-249.

Sanahuja, José Antonio (2012). «Post-liberal Regionalism in South America: The Case of UNASUR». Working Paper 05, Robert Schuman Centre for Advanced Studies.

Sanahuja, José Antonio (2007). «Regionalismo e integración en América Latina: 
balance y perspectivas». Pensamiento Iberoamericano 0(1): 75-106.

UNASUR. Consejo Suramericano de Economía y Finanzas (2011). Resumen del Consejo Suramericano de Economía y Finanzas desde su creación hasta 2011. Recuperado el 29 de mayo de 2014 de: http://docs.unasursg.org/

Unión Europea (2013, 27 de junio 2013). EUCELAC Action Plan 2013-2015. Recuperado de: http://data.consilium.europa.eu/ doc/document/ST-5748-2013-INIT/en/pdf
Van Klaveren, Alberto (1998). "América Latina: hacia un regionalismo abierto". Estudios Internacionales 117(30): 62-78.

Van Klaveren, Alberto (2011). «La política exterior de Chile durante los gobiernos de la Concertación (1990-2009)». Estudios Internacionales 169: 155-172.

Van Klaveren, Alberto (2012). «América Latina en un nuevo mundo». Revista CIDOB d'Afers Internacionals 100: 131-150.

Wilhelmy, Manfred \& Durán, Roberto (2003). «Los principales rasgos de la política exterior chilena entre 1973 y el 2000». Revista de Ciencia Política 23(2): 273-286. 